\title{
Expressions of Src homology 2 domain-containing phosphatase and its clinical significance in laryngeal carcinoma
}

\author{
L.B. Dong ${ }^{1}$, G.Q. Li ${ }^{2}$ Z.H. Tian' ${ }^{2}$ Z.M. Wang ${ }^{2}$ and K. Xu' \\ ${ }^{1}$ Department of Otolaryngology Head and Neck Surgery, \\ The 455th Hospital of Chinese PLA, Shanghai, China \\ ${ }^{2}$ Department of Pathology, The 455th Hospital of Chinese PLA, Shanghai, China \\ Corresponding author: L.B. Dong \\ E-mail: cco1965@163.com
}

Genet. Mol. Res. 12 (4): 4207-4212 (2013)

Received December 21, 2012

Accepted May 4, 2013

Published October 7, 2013

DOI http://dx.doi.org/10.4238/2013.October.7.6

\begin{abstract}
We investigated the expression of Src homology 2 domain-containing phosphatase (SHP-2) in laryngeal carcinoma and its clinical significance. Expression of SHP-2 was detected by immunohistochemical staining in normal mucosal tissues and various grades of laryngeal carcinoma. We looked for possible correlations between expression of SHP-2 in laryngeal carcinoma and clinical staging and lymph node metastasis. Immunochemical staining results revealed that the SHP-2 expression was significantly higher (88.24\%) in laryngeal carcinoma than in normal mucosal tissue (25\%). Additionally, the expression of SHP-2 was significantly correlated with lymph node metastasis, but not with clinical stage and gender of patients with laryngeal carcinoma. Therefore, SHP-2 may be useful as a prognostic marker for laryngeal carcinoma and as a therapeutic target in laryngeal carcinoma treatment.
\end{abstract}

Key words: Src homology 2 domain-containing phosphatase (SHP-2); Laryngeal carcinoma; Immunohistochemistry 


\section{INTRODUCTION}

Src homology 2 domain-containing phosphatase (SHP-2), an important protein-tyrosine phosphatase (PTP), acts as a downstream intracellular signal molecule for a number of extracellular stimuli, including cytokines and growth factors. SHP-2 is widely expressed in tissues and plays an important role in the signal transduction associated with cell proliferation and survival (Qu, 2000).

The correlation between the expression of SHP-2 and non-small cell lung cancer, leukemia and prostatic cancer has been identified in a number of studies worldwide (Tartaglia et al., 2003, 2004; Bentires-Alj et al., 2004; Loh et al., 2004b). It has also been demonstrated that the expression of SHP-2 is increased in breast cancer, leukemia and solid skin tumors. Further molecular biology research has identified the conspicuous correlation between SHP-2 expression and tumor presentation, and it is now a hot spot for clinical diagnosis and tumor treatment.

Laryngeal carcinoma (LC) is a common malignant tumor of the head and neck, which has serious effects on human health. To the best of our knowledge, the function of SHP-2 in the progression and development of LC has not been studied. Our study begins to research this point with the aim of exploring the importance of variation in the expression of SHP-2 in the development and morbidity of LC.

\section{MATERIAL AND METHODS}

\section{Source of specimens}

LC tissue samples were collected from 17 patients seen by our hospital's Hematology Department and wards during January 2008 to May 2011. None of the patients had received chemotherapy and radiotherapy before operations. Thirteen cases were males and the other 4 were females, and the average age of patients was 57.8 years. LC was classified according to the Union Internationale Contre le Cancer (UICC) TNM classification of 2010. The clinical stage of LC were characterized as follows: I, 2 cases; II, 3 cases; III, 5 cases; and IV, 7 cases. Lymph node metastasis was pathologically confirmed in 7 cases and in the rest not. All isolated specimens were handled within $2 \mathrm{~h}$; they were fixed in $10 \%$ neutral formalin for $24 \mathrm{~h}$, paraffin-embedded and hematoxylin and eosin (HE)-stained, and the pathological properties and grade were ascertained. All patients gave informed consent before participating, and the study was approved by the local research ethics committee.

\section{Reagents}

SHP-2 rabbit antibody was purchased from Santa Cruz Biotechnology, Inc. (Santa Cruz, CA, USA). The other reagents were conventional reagents from our office section.

\section{Histological and immunohistochemical staining}

The specimens were fixed and paraffin-embedded, and serial sections $(5 \mu \mathrm{m})$ were cut and submitted to HE staining and PTP antibody (anti-SHP-2) immunostaining according to the manufacturer instructions. The results were graded according to the scoring system used by 
Zhang et al. (2009). Cells stained with brown granules in the cytoplasm were graded as SHP2 positive. Thus, the degree of SHP-2 staining could be assessed using the intensity and the percentage of positively stained cells. The staining intensity was classified into four groups: 0 , unstained; 1 , weakly stained; 2, moderately stained; and 3, strongly stained. A total of 10 typical high-power fields were selected, and 100 cells were randomly counted to calculate the percentage of positively stained cells. The specimens were graded: 0 , for less than $5 \% ; 1$, for 5 to $25 \%$; 2 , for 25 to $50 \%$; and 3, for greater than $50 \%$. Subsequently, we multiplied the staining intensity score by the grade for percentage of positive cells. A results of 0-1 was defined as negative (-), 2-3 as weak positive $(+), 4-5$ as positive $(++)$, and 6 or greater as strong positive $(+++)$.

\section{Statistical analysis}

The statistical analysis was conducted using SPSS 10.0 (SPSS, Inc., Chicago, IL, USA) using one-way ANOVA. $\mathrm{P}<0.05$ indicated a statistically significant difference.

\section{RESULTS}

\section{Expression and distribution of SHP-2 in LC tissues}

In normal mucosa tissues, a pale yellow staining was observed in transitional epithelial cells, representing a low expression of SHP-2. However, in LC tissues a simple light or dark brown colored staining was observed in all pathological stages, representing a high expression of SHP-2 (Figure 1).
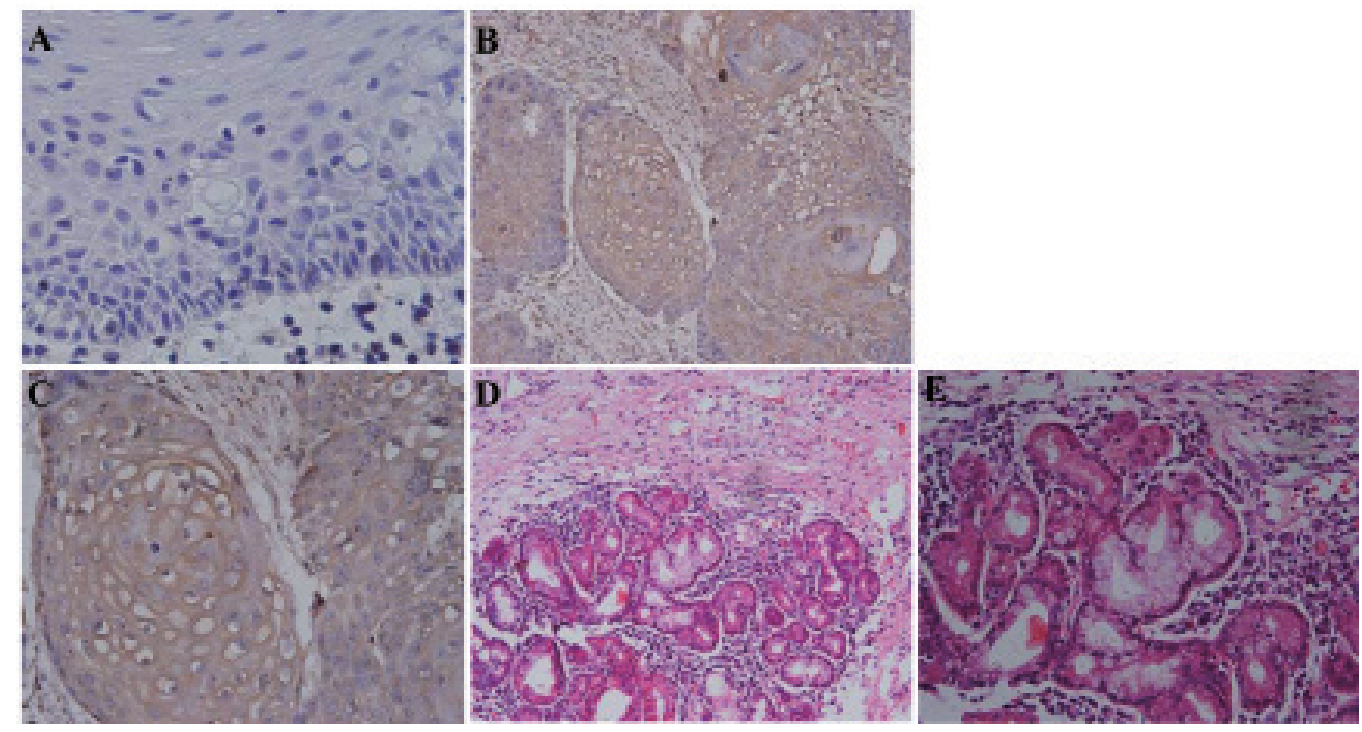

Figure 1. Histological and immunohistochemical staining detection of the expression and distribution of SHP-2 in laryngeal carcinoma (LC) tissues. A. Normal mucosa (400X magnification). B. and C. LC tissues (200X and 400X magnification, respectively). D. and E. LC tissues stained with hematoxylin and eosin (200X and 400X magnification, respectively). 


\section{Positive expression rate of SHP-2}

Among the 17 LC tissue samples, a total of 15 cases were SHP-2 positive, yielding a positive expression rate of $88.24 \%$. Among the 4 normal mucosa tissue samples, only 1 case displayed a positive expression of SHP-2, yielding a positive expression rate of $25 \%$. The variant expression rate of SHP-2 between LC_and normal mucosa tissues was analyzed using the Student $t$-test and the difference was considered to be statistically significant $(\mathrm{P}<0.05$; Table 1$)$.

Table 1. Expression of SHP-2 in laryngeal carcinoma and normal mucosal tissues.

\begin{tabular}{|c|c|c|c|c|c|c|c|}
\hline \multirow[t]{2}{*}{ Tissue } & \multirow[t]{2}{*}{ Number of cases } & \multicolumn{4}{|c|}{ Expression of SHP-2 } & \multirow{2}{*}{ Positive rate $(\%)$} & \multirow[t]{2}{*}{$P$ value } \\
\hline & & - & + & ++ & +++ & & \\
\hline Laryngeal carcinoma & 17 & 2 & 3 & 5 & 7 & 88.24 & $<0.05$ \\
\hline Normal mucosal tissue & 4 & 3 & 1 & 0 & 0 & 25 & \\
\hline
\end{tabular}

Compared with patients without lymph node metastasis, the positive expression rate of SHP-2 was significantly higher in tissues of patients with lymph node metastasis $(\mathrm{P}<0.05$; Table 2). Additionally, the positive expression of SHP-2 was not significantly correlated with clinical stage or gender of patients with LC $(\mathrm{P}>0.05$; Table 2$)$.

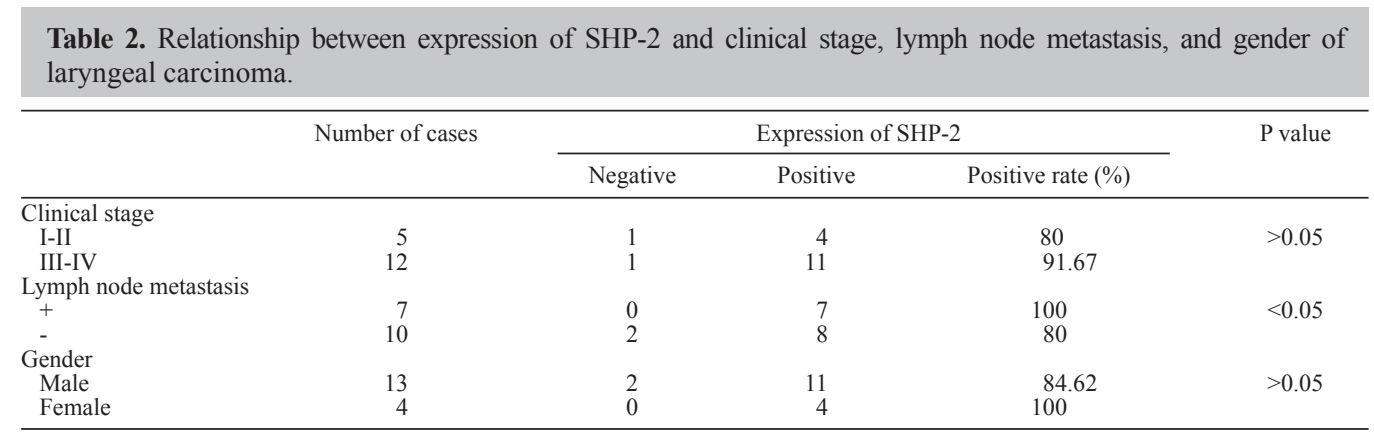

\section{Correlation between the expression of SHP-2 in LC tissues and the prognosis of patients}

Among the 17 LC cases studied, only 7 mortalities occurred, all of which demonstrated a positive SHP-2 expression. Therefore, the survival rate for the SHP-2 positive tissues was $53.33 \%(7 / 15)$. A $t$-test demonstrated that there was a marked difference between the two groups $(\mathrm{P}<0.05)$. The survival rate was significantly higher in patients with negative SHP-2 expression compared to patients with positive expression.

\section{DISCUSSION}

LC, a common malignancy in the head and neck region, presents a serious health threat. It accounts for approximately $1.2 \sim 1.6 \%$ of the total malignant tumor cases. At the same time, it contributes to approximately $7.9 \sim 35 \%$ of ear, nose and throat (ENT) cancers. Statistics have shown that the incidence of LC is approximately $4 / 100,000$ cases. The morbidity of LC 
varies greatly in different regions of the world.

In certain countries, the morbidity level is up to $17.7 / 100,000$ cases. Statistical reports have revealed that the incidence of LC is more common in urban areas compared to the countryside. In recent years, the morbidity of LC exhibited a rising trend as a result of the harmful effects caused by an increased exposure to various environmental carcinogenic factors. In reality, patients in stage III-IV, and those with recurrence or metastasis, do not respond well to existing treatments; thus, LC has become a malignant disease which is life-threatening for numerous individuals. With further research in molecular biology, the study of the correlation between tumors and SHP-2 is of particular interest and has become a significant factor in clinical diagnosis and oncotherapy (Qu et al., 1997; Guillemot et al., 2000; Su et al., 2001; Qu, 2002).

Complicated signal transduction pathways are involved in carcinogenesis and the growth and development of cells (Qu et al., 1998). A large number of signal molecules are implicated in these pathways, in which phosphorylation is a basic biochemical process. Studies have revealed that carcinogenesis increases in accordance with the rise in the levels of protein phosphorylation $(\mathrm{Qu}$ et al., 2001). Therefore, we conjectured that the protein phosphorylation level may be the key factor associated with the proliferation and differentiation mechanism of tumors. Additionally, the intracellular protein phosphorylation level is regulated by the mutual coordination of PTP and protein tyrosine kinase (PTK). Thus, it has been suggested that PTP is closely correlated with tumorigenesis. Studies have confirmed that SHP-1 and SHP-2 are first expressed in hematopoietic cells and lymphocytes. They play various roles in signal transduction as well as cell proliferation and differentiation; however, a disorder in cell proliferation and differentiation is a characterization of tumor cells.

The role of SHP-2 in signal transduction remains unclear; however, it may have certain upregulatory effects. It is possibility that the negative regulation of SHP-1 maintains a dynamic equilibrium with the positive regulation of SHP-2. Once the dynamic equilibrium is impaired, it would directly influence cell proliferation and signal transduction. The present study demonstrated that the expression of SHP-2 is significantly increased in LC tissues, with no marked signs of a shift in normal mucosa tissues. This suggests that when the normal transitional epithelial tissues near the LC undergo malignant transformation, the expression of SHP-2 significantly increases at the same time. Therefore, the expression of SHP-2 may be a clinical diagnostic index for the malignant transformation of transitional cells.

Our results revealed that the positive expression rate of SHP-2 is significantly higher in tissues of patients with lymph node metastasis compared with patients without lymph node metastasis, suggesting that the positive expression of SHP-2 is positively correlated with lymph node metastasis. However, further statistical analysis for the correlation between the expression of SHP-2 in LC and gender and clinical staging revealed that expression of SHP-2 was not significantly correlated with the clinical stage or gender of patients with LC. SHP-2, as an important PTP, is a vital molecule in cell proliferation and survival-related signal transduction (Henshall et al., 2001). Among those malignant tumors for example lung solid tumors, colon solid tumors, nervous system solid tumors and skin solid tumors, the activating mutations of SHP-2 is commonly found (Loh et al., 2004a).

Other studies have indicated that SHP-2 may be a useful target to regulate Bcr-Ab1-positive leukemia and chronic myeloid leukemia (Chen et al., 2007). Certain studies have revealed that SHP2 may play a positive role in regulating the receptor-mediated signaling pathway of receptor tyrosine kinase. Additionally, SHP-2 may also partially block signal transduction via dephosphorylation of signal transduction molecules (Charest et al., 2006). Although the function of SHP-2 in the progres- 
sion and development of laryngeal neoplasms has not been confirmed, there are a number of studies identifying its impact on other forms of tumorigenesis; for example, the association between changes in SHP-2 expression and non-small cell lung cancer, leukemia and prostate cancer (Lieskovska et al., 2006; Jamshidi et al., 2007). The present study tentatively explored the role of SHP-2 in LC, and it confirmed that the expression level of SHP-2 in LC tissues was significantly higher compared to that of normal tissues. In the future, the biological correlation between the increased expression of SHP-2 and cell proliferation and apoptosis in LC cells requires extensive studies.

\section{ACKNOWLEDGMENTS}

Research supported by the Scientific Research Project of the 455th Hospital of Chinese PLA (Grant \#01Z041).

\section{REFERENCES}

Bentires-Alj M, Paez JG, David FS, Keilhack H, et al. (2004). Activating mutations of the noonan syndrome-associated SHP2/PTPN11 gene in human solid tumors and adult acute myelogenous leukemia. Cancer Res. 64: 8816-8820.

Charest A, Wilker EW, McLaughlin ME, Lane K, et al. (2006). ROS fusion tyrosine kinase activates a SH2 domaincontaining phosphatase-2/phosphatidylinositol 3-kinase/mammalian target of rapamycin signaling axis to form glioblastoma in mice. Cancer Res. 66: 7473-7481.

Chen J, Yu WM, Daino H, Broxmeyer HE, et al. (2007). SHP-2 phosphatase is required for hematopoietic cell transformation by Bcr-Abl. Blood 109: 778-785.

Guillemot L, Levy A, Zhao ZJ, Bereziat G, et al. (2000). The protein-tyrosine phosphatase SHP-2 is required during angiotensin II-mediated activation of cyclin D1 promoter in CHO-AT1A cells. J. Biol. Chem. 275: 26349-26358.

Henshall TL, Jones KL, Wilkinson R and Jackson DE (2001). Src homology 2 domain-containing protein-tyrosine phosphatases, SHP-1 and SHP-2, are required for platelet endothelial cell adhesion molecule-1/CD31-mediated inhibitory signaling. J. Immunol. 166: 3098-3106.

Jamshidi Y, Gooljar SB, Snieder H, Wang X, et al. (2007). SHP-2 and PI3-kinase genes PTPN11 and PIK3R1 may influence serum apoB and LDL cholesterol levels in normal women. Atherosclerosis 194: e26-e33.

Lieskovska J, Ling Y, Badley-Clarke J and Clemmons DR (2006). The role of Src kinase in insulin-like growth factordependent mitogenic signaling in vascular smooth muscle cells. J. Biol. Chem. 281: 25041-25053.

Loh ML, Reynolds MG, Vattikuti S, Gerbing RB, et al. (2004a). PTPN11 mutations in pediatric patients with acute myeloid leukemia: results from the Children's Cancer Group. Leukemia 18: 1831-1834.

Loh ML, Vattikuti S, Schubbert S, Reynolds MG, et al. (2004b). Mutations in PTPN11 implicate the SHP-2 phosphatase in leukemogenesis. Blood 103: 2325-2331.

Qu CK (2000). The SHP-2 tyrosine phosphatase: signaling mechanisms and biological functions. Cell Res. 10: 279-288.

Qu CK (2002). Role of the SHP-2 tyrosine phosphatase in cytokine-induced signaling and cellular response. Biochim. Biophys. Acta 1592: 297-301.

Qu CK, Shi ZQ, Shen R, Tsai FY, et al. (1997). A deletion mutation in the SH2-N domain of Shp-2 severely suppresses hematopoietic cell development. Mol. Cell Biol. 17: 5499-5507.

Qu CK, Yu WM, Azzarelli B, Cooper S, et al. (1998). Biased suppression of hematopoiesis and multiple developmental defects in chimeric mice containing Shp-2 mutant cells. Mol. Cell Biol. 18: 6075-6082.

Qu CK, Nguyen S, Chen J and Feng GS (2001). Requirement of Shp-2 tyrosine phosphatase in lymphoid and hematopoietic cell development. Blood 97: 911-914.

Su MW, Yu CL, Burakoff SJ and Jin YJ (2001). Targeting Src homology 2 domain-containing tyrosine phosphatase (SHP1) into lipid rafts inhibits CD3-induced T cell activation. J. Immunol. 166: 3975-3982.

Tartaglia M, Niemeyer CM, Fragale A, Song X, et al. (2003). Somatic mutations in PTPN11 in juvenile myelomonocytic leukemia, myelodysplastic syndromes and acute myeloid leukemia. Nat. Genet. 34: 148-150.

Tartaglia M, Martinelli S, Cazzaniga G, Cordeddu V, et al. (2004). Genetic evidence for lineage-related and differentiation stagerelated contribution of somatic PTPN11 mutations to leukemogenesis in childhood acute leukemia. Blood 104: 307-313.

Zhang S, Chen LY and Li L (2009). Significance of Topoisomerase? in Primary Gastric Carcinomas. J. Fujian Med. Univ. 40: 132-134. 